\title{
Development of continuous processes for vegetable oil alcoholysis in microfluidic devices
}

\author{
Romain RICHARD ${ }^{1,2,3,4}$ \\ Brigitte DUBREUIL ${ }^{1,2}$ \\ Laurent PRAT ${ }^{3,4}$ \\ Sophie THIEBAUD-ROUX ${ }^{1,2}$ \\ 1 Université de Toulouse, \\ INP-ENSIACET, \\ LCA (Laboratoire de Chimie Agro- \\ industrielle), \\ F-31030 Toulouse, France \\ $<$ sophie.thiebaudroux@ensiacet.fr $>$ \\ 2 INRA, \\ UMR 1010 CAI, \\ F-31030 Toulouse, France \\ ${ }^{3}$ Université de Toulouse; \\ INP, UPS ; \\ Laboratoire de Génie Chimique; \\ 4 allée Emile Monso, \\ F-31030 Toulouse, France \\ ${ }^{4}$ CNRS; \\ Laboratoire de Génie Chimique; \\ F-31030 Toulouse, \\ France
}

Article received 18 September 2012

Accepted 26 September 2012

Biodiesel can be produced from vegetable oils, animal fats, and waste cooking oils by transesterification (Demirbas, Karslioglu, 2007; Encinar et al., 2007) with an alcohol in order to substitute fossil fuels. Many works have used methanol (Darnoko, Cheryan, 2000; Fukuda et al., 2001; Sharma, Singh, 2008; Srivastava, Verma, 2008) as alcohol reactant which is mainly produced by oxidation processes of methane, a natural gas component, hence a non-renewable energy. Ethanol and particularly bioethanol from sugar cane, sugar beet or corn is preferable to methanol due to

\begin{abstract}
Biodiesel can be produced from vegetable oils, animal fats, and waste cooking oils by transesterification with ethanol (also called ethanolysis) in order to substitute fossil fuels. In this work, we were interested in the transesterification reaction of sunflower oil with ethanol, which leads to ethyl esters, used to date for applications principally in food and cosmetic industry. To open the application field to biofuels (to substitute current fuels resulting from fossil resources), the process efficiency has to be developed to be economically profitable. The batch reaction of vegetable oil ethanolysis was transposed to a micro-scaled continuous device (PFA tube of $508 \mu \mathrm{m}$ internal diameter), inducing better heat and mass transfer. Study of the influence of the operational conditions (reactants flow, initial ethanol to oil molar ratio, temperature. . .) revealed the favourable reaction parameters necessary to reach high conversions and yields. In these conditions, it is possible to acquire kinetics data at the first seconds of the reaction, which was not feasible in a conventional batch process. These data were used to model occurring phenomena and to determine kinetic constants and transfer coefficients. The model was subsequently used to simulate reactions with other operational conditions. To acquire these data in microreactors, an on-line analysis method by Near InfraRed (NIR) spectroscopy was developed by using gas chromatography as a reference method. PLS models were then set up to quantify on-line the major compounds contents during the reaction.
\end{abstract}

Key words: biodiesel, ethanolysis, microreactors, NIR spectroscopy, modelling, simulation

its superior dissolving power for vegetable oils, low toxicity and its renewable origin. Various factors such as free fatty acid (FFA) content, water content, type/ amount of catalyst, vegetable oil to alcohol molar ratio, or temperature (Freedman et al., 1984; Darnoko, Cheryan, 2000; Meher et al., 2006) can affect the process. The oil transesterification using ethanol leads to high conversion of triglycerides into ethyl esters with diglycerides and monoglycerides as reaction intermediates and glycerol as by-product. Indeed, this reaction is composed of three consecutive steps (figure 1).
This reaction, also called ethanolysis, is generally conducted in batch reactors. Depending on the scheme and the thermokinetic properties of the system, continuous processes may withdraw existing obstacles of batch processes such as numerous steps, secondary reactions, stable equilibria and difficulties to separate the products. Several works on biodiesel production using microreactors have recently been published (Sun et al., 2008; Sun et al., 2009; Wen et al., 2009; Qiu et al., 2010). The ethanolysis system is complex due to changing phase equilibria (Guan et al.,

To cite this article: Richard R, Dubreuil B, Prat L, Thiebaud-Roux S. Development of continuous processes for vegetable oil alcoholysis in microfluidic devices. OCL 2013; 20(1): 23-32. doi : 10.1684/ocl.2012.0479 
2010): two immiscible phases are present at the beginning (sunflower oil and ethanol), then a single phase appears after a few minutes of reaction. Finally, two phases are obtained at the end of the reaction, majorly composed of ethyl esters and glycerol. Moreover, this reaction involves simultaneous and coupled phenomena (mixing, heat and mass transfers) which have to be precisely controlled. Hence, to properly design a continuous process, numerous data are required. In this work, we transferred the batch ethanolysis of high oleic sunflower oil into a continuous microstructured device, which induces a better control of heat and mass transfers. Various parameters have been studied, notably the initial ethanol to oil molar ratio. Furthermore, the acquired data ensure the modelling of chemical kinetics and mass transfer phenomena for the transesterification reaction. Kinetics constants and mass transfer coefficients were then calculated in order to match experimental data with modelling data. The model was subsequently used to simulate reactions with other operational conditions and to propose other process implementation.

Analytical procedures have been developed to determine the composition of crude transesterification products. In previous studies, different analytical procedures (gas chromatography (Mittelbach, 1993; Knothe, 2001), high performance liquid chromatography (Holcapek et al., 1999), Fourier transform infrared spectroscopy (Zagonel et al., 2004; Mahamuni, Adewuyi, 2009) have been developed to off-line monitor the composition of reaction mixtures obtained during or after transesterification of vegetable oils with ethanol containing also FFA, ethyl esters, monoglycerides, diglycerides, triglycerides and glycerol. Nevertheless, these techniques are long to handle, unreliable and expensive methods. Up to now, only a few techniques such as ultrasonicationassisted spray ionization mass spectroscopy (Chen et al., 2010) or mid-infrared spectroscopy (Trevisan et al., 2008) can on-line monitor organic reactions.

Faced to this lack of analysis techniques, we developed an innovative method using Near Infrared (NIR) spectroscopy to on-line monitor the transesterification reaction of high oleic sunflower oil with ethanol in microreactors (circular PFA tube $1 / 16^{\prime \prime}$ OD, $0.02^{\prime \prime}$ ID). NIR spec- troscopy with multivariate analysis is a well-established, fast, reliable, inexpensive and non-destructive analytical technique, and does not require complex pre-treatment (Knothe, 1999; Balabin et al., 2010; Richard et al., 2011). The reactions were monitored through sequential scans of the reaction medium with an adequate probe on microreactors. Partial Least Squares (PLS) regression was used to develop calibration models between NIR spectral data and analytical data obtained by a reference method (gas chromatography with flame ionization detection, GCFID). The developed models were validated by comparison with the GC data.

\section{Materials and methods}

\section{Material and reagent}

High oleic sunflower oil was obtained at ITERG (Pessac, France) from seeds produced by ARTERRIS (Toulouse, France). Its fatty acid composition (\% by weight) is as follows: $3.5 \%$ palmitic acid, $3.0 \%$ stearic acid, $87.6 \%$ oleic acid, $4.9 \%$ linoleic acid and $1.2 \%$ other acids. Hydrochloric acid (analytical quality, 3,7\% solution in water) and sodium ethoxide EtONa (analytically pure, $21 \mathrm{wt} \%$ in ethanol) were purchased from Acros Organics; absolute ethanol (99.95\%) and cyclohexane (HPLC grade) were supplied by Scharlau. N-methyl$\mathrm{N}$-trimethylsilyl-heptafluorobutyramide (MSHFBA) and methylimidazole (MI) were purchased respectively from Macherey Nagel and Sigma-Aldrich.

\section{Transesterification reaction}

The transesterification reaction was performed in circular PFA tubes (1/ $\left.16^{\prime \prime} \mathrm{OD}, 0.02^{\prime \prime} I D\right)$. These tubes were dipped in a temperature-controlled water bath to maintain the targeted microreactor temperature $\left(65^{\circ} \mathrm{C}\right.$, close to the boiling point of ethanol). Transesterification of high oleic sunflower oil was carried out by using the following procedure: liquids (oil and EtOH/EtONa mixture) were injected by syringepumps with perfect flow control. Several ethanol to oil molar ratios have been tested (45.4, 22.7, 16.2, 9.0 and 6.0) with the same amount of catalyst (1 wt $\%$ compared to oil mass). After mixing in a T-junction, the medium flows into the tube (figure 2). With constant flows, each length of tube corresponds to a reaction time. We used a $2 \mathrm{~m}$-length tube which corresponds approximately to a reaction time of $16 \mathrm{~min} \quad\left(\mathrm{Q}_{\text {tot }}=1.5 \mathrm{~mL} \cdot \mathrm{h}^{-1}\right.$ and $\mathrm{U}_{\text {tot }}=2.12 \mathrm{~mm} \cdot \mathrm{s}^{-1}$ ). The tube was cut in order to obtain lower reaction times. Samples were collected during $15 \mathrm{~min}$ with a concomitant addition of a continuous $\mathrm{HCl}$ flow $(3.7 \%$ solution in water) in order to quench the reaction by neutralizing the alkali catalyst. For each sample, the medium composition at different reaction times was determined by using GC-FID analysis. In the meanwhile, NIR spectroscopy was performed at the same reaction time as the sample collection in order to on-line monitor the transesterification reaction.

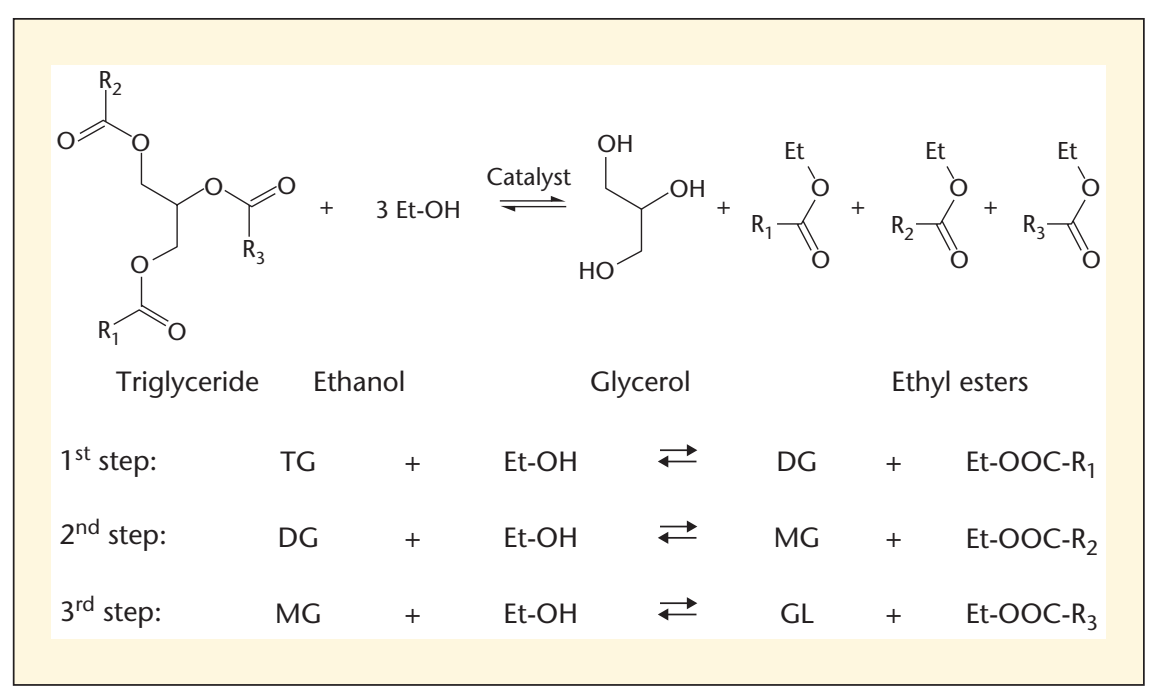

Figure 1. Global transesterification reaction and its three consecutive steps (where $R_{1}, R_{2}, R_{3}$ are fatty chains). 


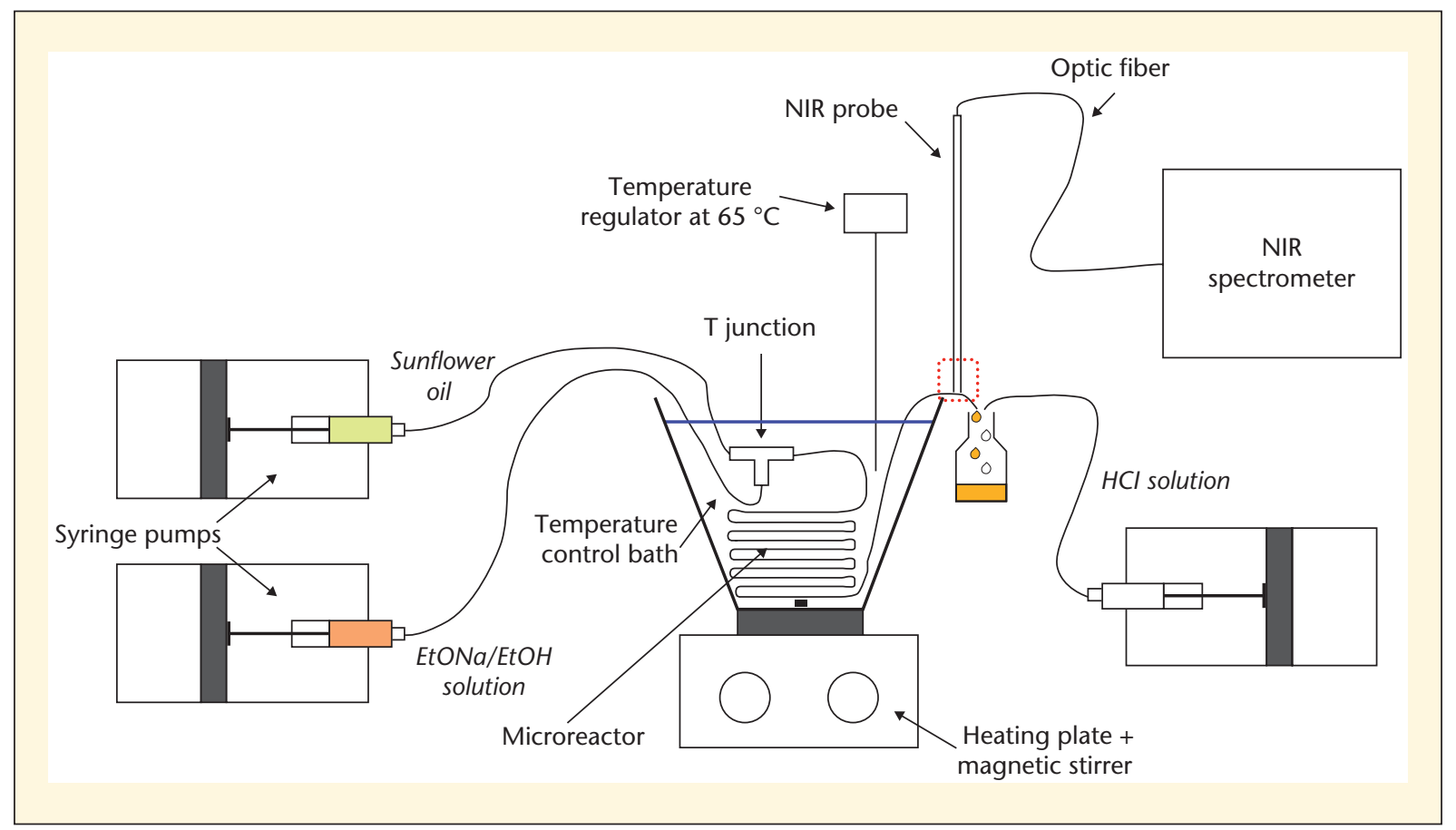

Figure 2. Experimental set-up of the transesterification reaction carried out in a microreactor.

\section{Gas chromatography analysis}

The collected samples were analyzed by gas chromatography using a Perkin Elmer Instrument (Perkin Elmer, USA) coupled to a flame ionization detector (FID). Separation was carried out with a silica capillary column (CP-Sil 8 CB lowbleed/MS, 5\% phenyl + 95\% dimethylpolysiloxane, $15 \mathrm{~m}, 0.32 \mathrm{~mm}$, $0.25 \mu \mathrm{m}$ ) from Varian (USA). The chromatograph was equipped with an automatic injector and the injections $(1 \mu \mathrm{L})$ were performed with an "oncolumn" injector.

All the samples were previously silylated with a mixture of MSHFBA and MI. This hydroxyl group silylation is intended to increase volatility and stability of the hydroxy compounds injected, and therefore to improve their detection. Cyclohexane was used as solvent to prepare standard solutions and heptadecane was used as the internal standard.

With this analytical procedure, molar concentrations of ethyl esters, oleic acid, monoglycerides, diglycerides and triglycerides in the different phases resulting from the reaction are determined. The gas chromatography data was used to calculate the total ethyl ester (more precisely ethyl oleate) content in oleic chains mixture (Eq.1):

Ethyl Ester Content (\%) =

$[E E]_{t}$

$[E E]_{t}+\left[C E_{18: 1}\right]_{t}+[M G]_{t}+2 \times[D G]_{t}+3 \times[T G]_{t}$

where $[E E]_{t},\left[C_{18: 1}\right]_{t},[M G]_{t},[D G]_{t}$ and $[\mathrm{TG}]_{\mathrm{t}}$ represent respectively the molar concentrations $(\mu \mathrm{mol} / \mathrm{ml})$ of ethyl oleate, oleic acid and oleic monoglyceride, diglyceride and triglyceride at a given time $\mathrm{t}$.

\section{NIR spectroscopy analysis}

The NIR spectra of the transesterification reaction mixture samples were recorded by averaging 32 scans (approximately $1 \mathrm{~s} / \mathrm{scan}$ ) in the wavelength range of $10,000-4000 \mathrm{~cm}^{-1}$ using a NIR spectrometer (Antaris MX FT-NIR Process Analyzer from Thermo Fisher Scientific, USA). This apparatus could be equipped with two different fiber-optic probes. One works by reflection (figure $3 a$ ) whereas the other works by transflectance (figure $3 b$ ), which means that the beam passes through the PFA tube and the solution, reflects on a mirror, before passing again through the solution and the PFA tube. The optical beam path was $3 \mathrm{~mm}$ (twice the tube diameter) and the spectral resolution was $2 \mathrm{~cm}^{-1}$. The spectrometer was operated by Omnic (Thermo Fisher Scientific, USA) software. Before statistical analysis, the NIR spectrum of the empty PFA tube (without sample) has been subtracted from the NIR spectra of the transesterification reaction mixture.

\section{Statistical analysis}

TQ Analyst (Thermo Fisher Scientific, USA) software was used to import and analyze the data. Each NIR spectrum corresponds to a reaction aliquot which was collected and analyzed by GC-FID. The NIR analyses were not performed on the total wavelength range of NIR spectra $\left(10,000-4000 \mathrm{~cm}^{-1}\right)$ because several regions were not useful for data treatment. Thus, to improve the results of the statistical treatment, three different spectral regions were suggested to work by TQ analyst software from 8649.17 to $8084.57 \mathrm{~cm}^{-1}$, from 7096.76 to $5507.7 \mathrm{~cm}^{-1}$ and from 5049.55 to $4476.07 \mathrm{~cm}^{-1}$ because these regions are the most representative of the spectral variations.

A series of ethanol to oil molar ratios (from 6.0 to 45.4 ) were tested in the 
A

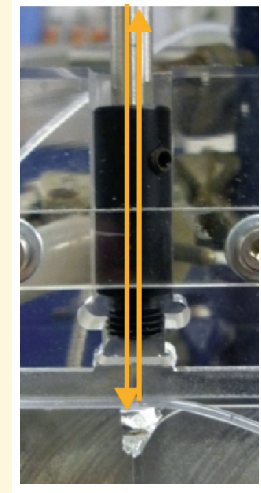

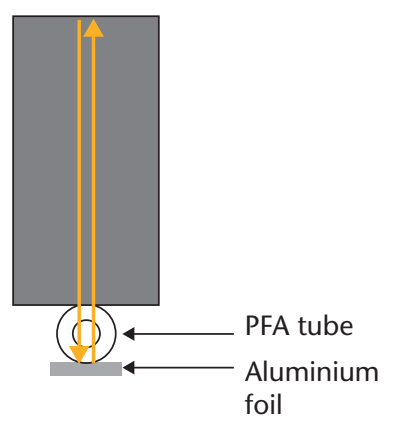

B

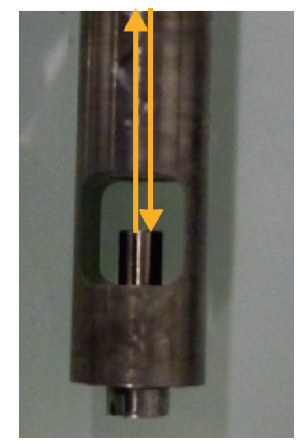

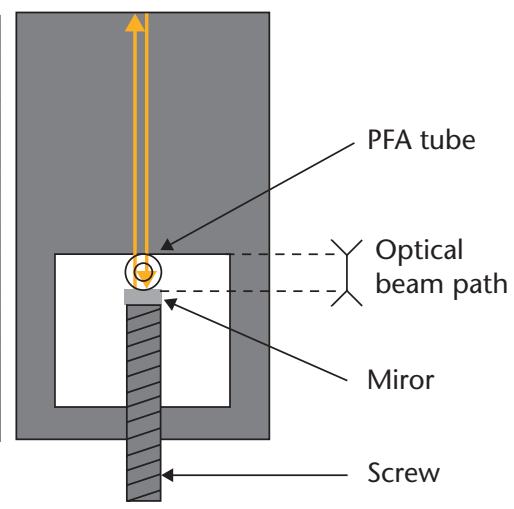

Figure 3. Pictures and schemes of the A) reflection probe and B) transflectance probe.

transesterification reaction at $65^{\circ} \mathrm{C}$. Samples were collected at different reaction times (from $47 \mathrm{~s}$ to $16 \mathrm{~min}$ ). Moreover, the experiment at a molar ratio of 45.4 was repeated 5 times. Thus a total of 130 samples were available for the data treatment.

Partial Least Squares (PLS) regression was used to develop calibration models between spectral and analytical GC data. This method is quantitative. The values of ethyl oleate contents were used to establish a multivariate model with the NIR spectra. In order to remove small inter-microreactor differences due to uncontrolled sources of variation, data pre-processing methods were applied to the spectra, such as baseline correction, Savitzky-Golay smoothing, first derivative, mean centering, variable scaling, standard normal variate (SNV) and also variables selection. Root Mean Square Error on Calibration (RMSEC) and Root Mean Square Error on Prediction (RMSEP) were used to evaluate the performance of the method. The closer the RMSE value is to zero, the smaller are the differences between the calculated concentrations values and the actual values.

From PLS regression, two calibration models have been developed. The first model is able to predict variations of the ethyl ester content in the reaction mixtures with varying ethanol to oil molar ratios from 6.0 to 45.4 . We used 69 samples for this model: 44 for calibration and 25 for validation. Be- sides, an other calibration model was developed from the spectra of the set of 61 samples (40 for the calibration and 21 for the external validation) all measured at $65^{\circ} \mathrm{C}$ for an ethanol to oil molar ratio of 45.4 to predict the contents of the two main chemical components: ethyl ester and oleic monoglycerides.

\section{Results and discussion}

\section{Hydrodynamic study}

First of all, effect of temperature on the flow inside microreactors was qualitatively studied. At room temperature, initial flow was constituted of droplets of oil in ethanol phase (figure 4). When the temperature was increased from $20^{\circ} \mathrm{C}$ to a limit temperature $\mathrm{T}_{\mathrm{L}}=45^{\circ} \mathrm{C}$, initial droplet flow was maintained and the reaction system becomes monophasic after several minutes of reaction, depending on the temperature. Above $T_{L}$, flow is constituted of two concentric jets which are initially stable, and then, as the reaction occurs, the system also became monophasic (after $1 \mathrm{~min}$ of reaction). Thus, dimensionless numbers of the system (Reynolds number Re and capillary number $\mathrm{Ca}$ ) were calculated for the two phases: the dispersed phase (index $d$, oil) and the continuous phase (index c, EtONa/EtOH mixture which was considered as of pure ethanol phase for calculations, weight concentration of EtONa in $\mathrm{EtOH}$ being very low of $0.42 \%)$.

Capillary number of the dispersed phase (oil) decreased with increasing temperature. Indeed, viscosity decreased, which means that viscous forces were lower and as surface tension was almost constant, capillary number decreased. For example, from $25^{\circ} \mathrm{C}$ to $65^{\circ} \mathrm{C}$, oil viscosity decreased by $74 \%$ while the surface tension decreased by $13.1 \%$. Above $T_{L}$, i.e. a limit capillary number $\left(\left(C a_{d}\right)_{L}=5.91 \times 10^{-4}\right)$, flow changed: it was no longer a droplet flow but a jet flow. Moreover, flow was laminar as Reynolds numbers calculated for each phase were respectively about $10^{-3}$ and $10^{-1}$ for the dispersed and continuous phases.

\section{Continuous transesterification processes in microreactors}

In batch processes, an ethanol to oil molar ratio of 6.0 seems to be optimal. It can be noticed that all the reactions reach equilibrium after about $20 \mathrm{~min}$ (Richard et al., 2011). Nevertheless, only few information on kinetics are available for low reaction times. In order to get more data as soon as the reaction starts, this reaction was carried out in a continuous system such as microreactors to acquire more data. In the case of the reaction mixtures with varying ethanol to oil molar ratio from 6.0 to 45.4 in microreactors, the reflection probe 


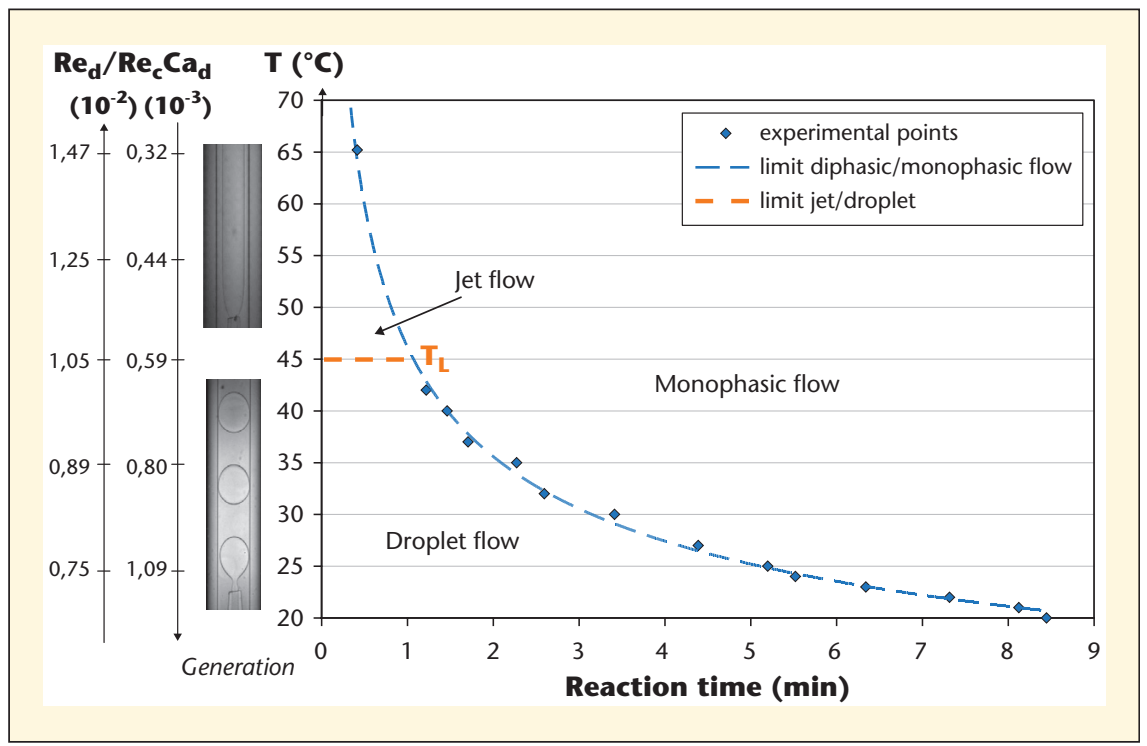

Figure 4. Influence of temperature on the flow in PFA tube $\left(F_{\text {EtOH/EtONal }} / F_{\text {oil }}=45.4\left(\mathrm{~mol}_{\mathrm{mol}}{ }^{-1}\right)\right.$, $\left.F_{\text {EtONa }} F_{\text {oil }}=1 \%\left(g \cdot g^{-1}\right), Q_{\text {tot }}=1.5 \mathrm{~mL} \cdot h^{-1}\right)$. was used to collect the NIR data. The total ethyl ester contents obtained by GC-FID according to the reaction time were illustrated in figure 5 . The highest molar ratios (16.2 to 45.4 ) accelerate the reaction rate at the beginning of the reaction.

With constant ethanol to oil molar ratio of 45.4, the NIR data were collected with the transflectance probe in order to show the repeatability of the experiment (5 times).

\section{Modelling of the kinetics: determination of a set of} parameters for each molar ratio

To build a model able to represent chemical kinetics and mass transfer phenomena, several hypotheses were formulated: (i) $1^{\text {st }}$ order for all compounds (and the catalyst) as results obtained for $1^{\text {st }}$ and $2^{\text {nd }}$ order are not significantly different (Richard et al., 2011); (ii) biphasic medium constituted by two phases (jet flows) such as an oil

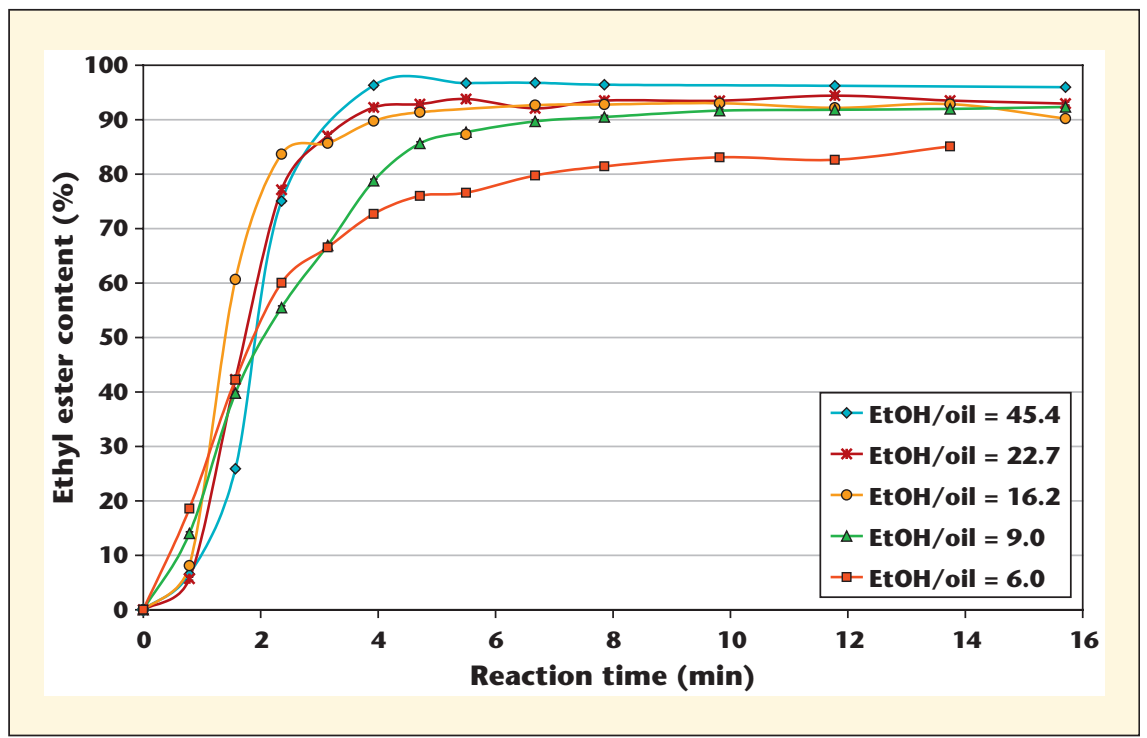

Figure 5. Total ethyl ester in the reaction mixtures at $65^{\circ} \mathrm{C}$ according to the reaction time for various ethanol to oil molar ratios in microreactors. phase (index $\mathrm{O}$ ) and an ethanol phase (index E): we suppose the transfer of oil (TG) as well as reaction intermediates (DG and MG) from the oil phase towards the ethanol phase (Poljanšek, Likozar, 2011); (iii) reaction in ethanol phase: the homogeneous catalyst being soluble in ethanol phase, the reaction takes place in this phase after the transfer of the species. The reaction kinetics is accelerated for a high amount of catalyst, which involves a faster conversion of triglycerides and thus an increase of their transfer rate (Stamenkovic et al., 2011); (v) balanced reactions: as the reactions are not total, TG, DG and MG are present at chemical equilibrium; (vi) no secondary reactions with free fatty acids (such as oleic acid): to simplify the model, we considered that reactions of salification of fatty acids and saponification of triglycerides and esters in presence of water were very fast and therefore insignificant compared to the others; (vii) as a consequence of hypothesis (vi), a pseudo-component was created: EE concentration is the sum of ethyl oleate and oleic acid concentrations.

Thanks to all these hypotheses, equations of the model representing mass transfer and $1^{\text {st }}$ order reaction kinetics for all the compounds can be written. For example, Eq.2-3 represent the TG concentration in ethanol and oil phases:

$$
\begin{aligned}
& \frac{d[T G]_{E}}{d t}=-k_{1}[T G]_{E}[E t O H][C a t a]+k_{-1}[D G]_{E}[E E] \\
& \times[C a t a]+k_{L} a_{(T C)}\left([T G]_{O}-[T G]_{E}\right) \\
& \frac{d[T G]_{O}}{d t}=-k_{L} a_{(T G)}\left([T G]_{O}-[T G]_{E}\right)
\end{aligned}
$$

Using a $1 \mathrm{~s}$ step time, the model was solved with a first order discretization. A parameter identification on kinetic constants and mass transfer coefficients can be carried out minimizing a criterion defined by the sum of the relative squared differences between the experimental measured data and the model output for each component of the reaction mixture (TG, DG, MG and EE) at different reaction times (Eq.4):

Minimization criterion $=\sum_{i} \sum_{i}\left(\frac{C_{j}(i)_{\bmod }-C_{j}(i)_{\exp }}{C_{j}(i)_{\exp }}\right)^{2}$

where $\mathrm{C}_{\mathrm{j}}(\mathrm{i})_{\text {mod }}$ is the calculated concentration of component $j$ at reaction time $i$, $\mathrm{C}_{\mathrm{j}}(\mathrm{i})_{\exp }$ is the experimental concentration (GC-FID data) of component $j$ at reaction time $i$ with $j=T G, D G, M G, E E$ 
and $i=47 s, 94 s, 141$ s, 189 s, $236 s$, 283 s, 330 s, 401 s, 471 s, 589 s, 707 s, $825 \mathrm{~s}, 942 \mathrm{~s}$. The minimization criterion on the relative squared variations was carried out separately for each molar ratio by using kinetic constants and mass transfer coefficients as variable parameters. For example, corresponding curves to an ethanol to oil molar ratio of 6.0 are represented on figure 6.

It should be pointed out that the model enables the representation of also the evolution of glycerol concentration as a function of reaction time, in addition to the concentration of the other components. The results of the parameters identification for the ethanol to oil molar ratio of 6.0 are gathered in table 1 . Results for the molar ratio 6.0 are satisfactory visually and numerically since the model curves really fit the experimental data for all the components and the total sum of their relative variations $\left(\Sigma \Sigma_{(\mathrm{j})}\right)$ for all reaction times is low (4.846). Kinetic constants are all of the same order of magnitude (between 1 and $10^{-2} \mathrm{~L}^{2} / \mathrm{mol}^{2} \mathrm{~s}$ ) except $\mathrm{k}_{-1}$ which is very low (about $10^{-10} \mathrm{~L}^{2} / \mathrm{mol}^{2} \mathrm{~s}$ ).

Parameters identification has been lead separately for each ethanol to oil molar ratio (Richard et al., 2012b) and results are also given in table 1 . Although $\Sigma_{(\mathrm{TG})}$ are quite high for ethanol to oil molar ratios 16.2 and 22.7, concentrations tendencies are correctly represented. Indeed, as concentrations of TG are very low, small variations can generate high relative errors.

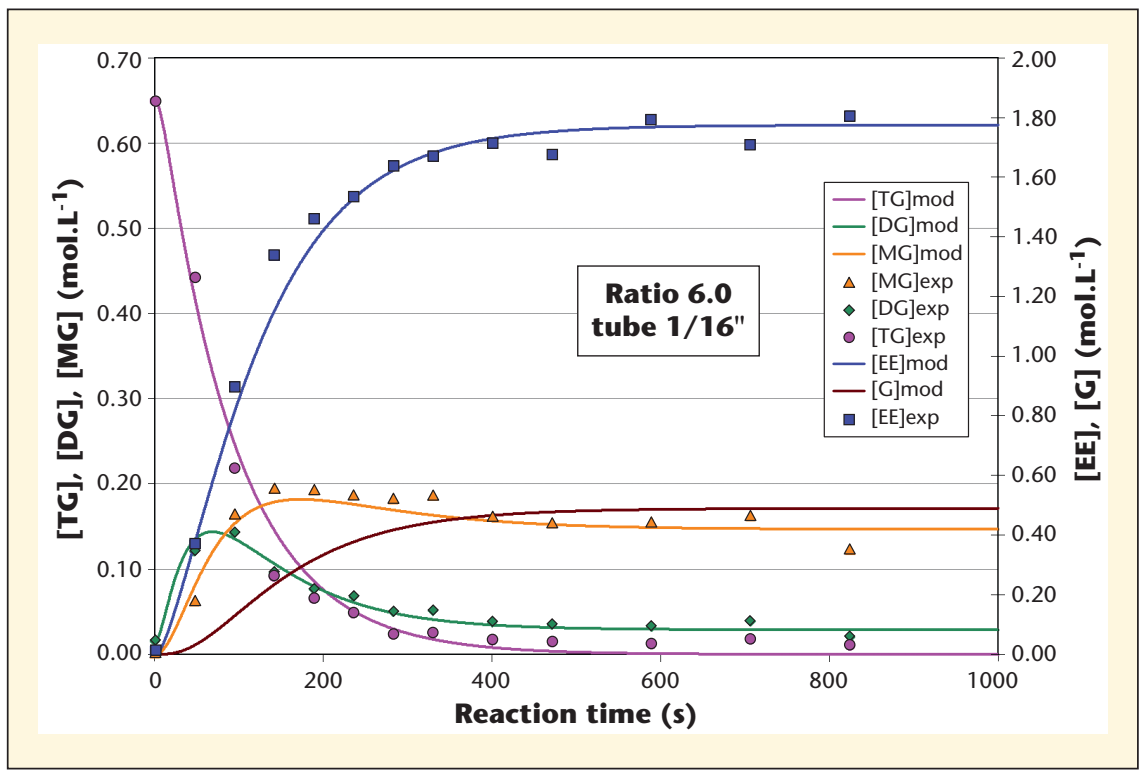

Figure 6. Evolution of the concentrations of the various oleic compounds according to the reaction time for a molar ratio of 6.0 (continuous lines are used for the model and singular points for the experimental data).

\section{Determination of a global set of parameters}

After determining the kinetic parameters (reaction rate constants and mass transfer coefficients) for each ethanol to oil molar ratio, we chose to establish common parameters able to correctly represent all the molar ratios used. First of all, the system was optimized using different initialization parameters and particularly the values previously determined for each molar ratio. In this case, the criterion to be minimized is the sum of the sums of the relative squared variations of each component at each reaction time for all molar ratios $\left(\Sigma \Sigma \Sigma_{\text {(ratios) }}\right)$.

Depending on the initializing parameters (different sets of kinetic and mass transfer parameters established for each molar ratio), the resulting criterion and then parameters can be different. The final value of the minimization criterion $\left(\Sigma \Sigma \Sigma_{\text {(ratios) }}\right)$ varies between 107.984 and 155.101 , which is mathematically ac-

Table 1. Results of the parameters identification carried out on 9 parameters by minimizing separately the criterion of the relative squared variations for each ethanol to oil molar ratio.

\begin{tabular}{|c|c|c|c|c|c|c|}
\hline Parameters & & 6.0 & 9.0 & 16.2 & 22.7 & 45.4 \\
\hline \multirow{6}{*}{ Kinetic constants } & $\mathrm{k}_{1}\left(\mathrm{~L}^{2} / \mathrm{mol}^{2} \mathrm{~s}\right)$ & $2.454 \times 10^{-1}$ & $1.000 \times 10^{-1}$ & $1.000 \times 10^{-1}$ & $1.454 \times 10^{-1}$ & $1.454 \times 10^{-1}$ \\
\hline & $\overline{\mathrm{k}_{-1}\left(\mathrm{~L}^{2} / \mathrm{mol}^{2} \mathrm{~s}\right)}$ & $8.649 \times 10^{-11}$ & $8.649 \times 10^{-11}$ & $8.649 \times 10^{-11}$ & $8.649 \times 10^{-11}$ & $8.649 \times 10^{-11}$ \\
\hline & $\mathrm{k}_{2}\left(\mathrm{~L}^{2} / \mathrm{mol}^{2} \mathrm{~s}\right)$ & $1.222 \times 10^{-1}$ & $1.087 \times 10^{-1}$ & $1.703 \times 10^{-1}$ & $2.000 \times 10^{-1}$ & $2.000 \times 10^{-1}$ \\
\hline & $\mathrm{k}_{-2}\left(\mathrm{~L}^{2} / \mathrm{mol}^{2} \mathrm{~s}\right)$ & $3.745 \times 10^{-2}$ & $1.225 \times 10^{-2}$ & $2.450 \times 10^{-2}$ & $3.745 \times 10^{-2}$ & $3.745 \times 10^{-2}$ \\
\hline & $\mathrm{k}_{3}\left(\mathrm{~L}^{2} / \mathrm{mol}^{2} \mathrm{~s}\right)$ & $5.367 \times 10^{-2}$ & $4.500 \times 10^{-2}$ & $5.367 \times 10^{-2}$ & $8.000 \times 10^{-2}$ & $8.000 \times 10^{-2}$ \\
\hline & $\overline{\mathrm{k}_{-3}\left(\mathrm{~L}^{2} / \mathrm{mol}^{2} \mathrm{~s}\right)}$ & $1.259 \times 10^{-2}$ & $9.205 \times 10^{-3}$ & $1.259 \times 10^{-2}$ & $3.000 \times 10^{-2}$ & $3.000 \times 10^{-2}$ \\
\hline \multirow{3}{*}{ Mass transfer coefficients } & $\mathrm{k}_{\mathrm{L}(\mathrm{TG})}(\mathrm{m} / \mathrm{s})$ & $1.502 \times 10^{-6}$ & $1.822 \times 10^{-6}$ & $2.000 \times 10^{-6}$ & $1.002 \times 10^{-6}$ & $1.002 \times 10^{-6}$ \\
\hline & $\mathrm{k}_{\mathrm{L}(\mathrm{DG})}(\mathrm{m} / \mathrm{s})$ & $1.763 \times 10^{-5}$ & $1.920 \times 10^{-5}$ & $4.687 \times 10^{-6}$ & $1.763 \times 10^{-5}$ & $1.763 \times 10^{-5}$ \\
\hline & $\overline{\mathrm{k}_{\mathrm{L}(\mathrm{MG})}(\mathrm{m} / \mathrm{s})}$ & $1.537 \times 10^{-6}$ & $6.331 \times 10^{-7}$ & $1.748 \times 10^{-5}$ & $1.537 \times 10^{-6}$ & $1.537 \times 10^{-6}$ \\
\hline \multirow{5}{*}{ Relative squared variations } & $\Sigma_{(\mathrm{EE})}$ & 0.039 & 0.008 & 1.641 & 2.504 & 1.240 \\
\hline & $\overline{\Sigma_{(M G)}}$ & 0.161 & 0.509 & 50.202 & 8.208 & 6.518 \\
\hline & $\overline{\Sigma_{(\mathrm{DG})}}$ & 0.358 & 1.171 & 6.168 & 11.130 & 0.482 \\
\hline & $\overline{\Sigma_{(\mathrm{TG})}}$ & 4.288 & 7.216 & 373.282 & 714.682 & 6.113 \\
\hline & $\overline{\Sigma \Sigma_{((\mathrm{j})}}$ & 4.846 & 8.904 & 431.293 & 736.525 & 14.356 \\
\hline
\end{tabular}


ceptable. However, although the modelling curves correctly fit to the experimental concentrations for ethyl ester whatever the molar ratio used, the representation of other compounds are less acceptable. Then, to improve the representation, we decided to manually determine a set of parameters able to correctly represent the evolutions of all the components concentrations for all molar ratios used. A global parameters identification minimizing criterion $\Sigma \Sigma \Sigma_{\text {(ratios) }}$ has been lead and the set of established parameters (kinetics constants and mass transfer coefficients) gives a criterion of 1165.406 (table 2).

Even if this total sum of the sums of the relative squared variations is much higher (1165.406) than the previous values (from 107.984 to 155.101 ), it is essentially due to the high value of the sum of the relative squared variations for the molar ratio of 16.2 (944.755) which represents $81 \%$ of the total sum. This important sum can be explained primarily by the relative squared variations obtained on TG, which represents a maximum of $96 \%$ of the total sum for molar ratio 16.2 , because of the rapid progression of TG concentrations towards values close to zero. Thus, corresponding graphs to this set of parameters, satisfactory for the whole of molar ratios used, are presented on figure 7.

\section{Using the model to simulate separation: examples of glycerol removals}

The model is useful to simulate other operating conditions such as, for example, glycerol removal. Indeed, it is a byproduct of the reaction and its removal shifts the equilibrium towards products formation in order to maximize ethyl ester formation (figure 1). Two different cases were studied. First of all, a total glycerol removal from a removal time $t_{R}$ until the end of the reaction was simulated for a transesterification reaction with an ethanol to oil molar ratio of 6.0 in a $1 / 16^{\prime \prime}$ PFA tube at $65^{\circ} \mathrm{C}$. This separation is equivalent to a decantation in a batch process. Figure $8 a$ illustrates a total glycerol removal $\left(\mathrm{F}_{\mathrm{G}(\mathrm{R})}=0.5 \mathrm{~mol}\right.$. $\mathrm{s}^{-1}$ ) from a reaction time $t_{\mathrm{R} 1}=500 \mathrm{~s}$.

From $t_{R 1}$, a glycerol removal was set with a sufficient glycerol molar flux to eliminate the totality of glycerol present in the reaction medium. For $t>t_{R}$,

Table 2. Results of global parameters identification with global manual adjustement.

\begin{tabular}{|ll|}
\hline Parameters & Global manual adjustement \\
\hline $\mathrm{k}_{1}\left(\mathrm{~mol}^{-2} \cdot \mathrm{L}^{2} \cdot \mathrm{s}^{-1}\right)$ & $1.200 \times 10^{-1}$ \\
\hline $\mathrm{k}_{-1}\left(\mathrm{~mol}^{-2} \cdot \mathrm{L}^{2} \cdot \mathrm{s}^{-1}\right)$ & $8.649 \times 10^{-11}$ \\
\hline $\mathrm{k}_{2}\left(\mathrm{~mol}^{-2} \cdot \mathrm{L}^{2} \cdot \mathrm{s}^{-1}\right)$ & $1.500 \times 10^{-1}$ \\
\hline $\mathrm{k}_{-2}\left(\mathrm{~mol}^{-2} \cdot \mathrm{L}^{2} \cdot \mathrm{s}^{-1}\right)$ & $3.000 \times 10^{-2}$ \\
\hline $\mathrm{k}_{3}\left(\mathrm{~mol}^{-2} \cdot \mathrm{L}^{2} \cdot \mathrm{s}^{-1}\right)$ & $5.000 \times 10^{-2}$ \\
\hline $\mathrm{k}_{-3}\left(\mathrm{~mol}^{-2} \cdot \mathrm{L}^{2} \cdot \mathrm{s}^{-1}\right)$ & $1.200 \times 10^{-2}$ \\
\hline $\mathrm{k}_{\mathrm{L}(\mathrm{TG})}\left(\mathrm{m} \cdot \mathrm{s}^{-1}\right)$ & $1.500 \times 10^{-6}$ \\
\hline $\mathrm{k}_{\mathrm{L} \text { (DG) }}\left(\mathrm{m} \cdot \mathrm{s}^{-1}\right)$ & $1.700 \times 10^{-5}$ \\
\hline $\mathrm{k}_{\mathrm{L} \text { (MG) }}\left(\mathrm{m} \cdot \mathrm{s}^{-1}\right)$ & $1.500 \times 10^{-6}$ \\
\hline$\Sigma \Sigma_{\text {(ratio } 6.0)}$ & 7.482 \\
\hline$\Sigma \Sigma_{\text {(ratio } 9.0)}$ & 26.580 \\
\hline$\Sigma \Sigma_{\text {(ratio } 16.2)}$ & 944.755 \\
\hline$\Sigma \Sigma_{\text {(ratio } 22.7)}$ & 160.959 \\
\hline$\Sigma \Sigma_{\text {(ratio } 45.4)}$ & 25.631 \\
\hline$\Sigma \Sigma_{\text {(ratios) }}$ & 1165.406 \\
\hline
\end{tabular}

glycerol was removed while forming (figure 8a). Oleic diglyceride and monoglyceride concentrations decrease while ethyl ester concentration increases. This is due to the equilibrium shift towards consumption of reaction intermediates and thus, the system tends to the maximum ethyl ester concentration $\left([\mathrm{EE}]_{\max }=1.98 \mathrm{~mol} \cdot \mathrm{L}^{-1}\right)$. Maximum yield of $100 \%$ could be reached with such a system.

Moreover, a constant and continuous glycerol removal (i.e. a glycerol-removing flux $\mathrm{F}_{\mathrm{G}(\mathrm{R})}$ in $\mathrm{mol}^{-1} \mathrm{~s}^{-1}$ ) starting after a removal time $t_{R 2}$ can be simulated. In the case presented in figure $8 b$, the glycerol removal flux is higher than glycerol formation flux, then glycerol concentration decreases continuously from $t_{R 2}$ to $t_{G=0}=972 \mathrm{~s}$, when glycerol concentration becomes null. The goal of continuous glycerol removal is to shift equilibrium, as in previous case, in order to form a maximum of ethyl esters. If the main objective is to reach a certain glycerol removal or to minimize the removal time, a parameter optimisation on glycerol flux and/or removal time, for example, has to be carried out. Obviously, the simulator is usable for other objectives.

\section{Partial Least Squares regression (PLS)}

Concerning the PLS regressions, only ethyl ester content has been predicted because it is the main compound of the medium as soon as the reaction starts. PLS regressions on other compounds such as monoglycerides, diglycerides and triglycerides contents were not performed because their concentration ranges were too limited to establish a valuable predicting model. Five latent variables have been used to build the PLS model for predicting ethyl oleate contents of the reaction mixture obtained with different ethanol to oil molar ratios. The predicted contents of ethyl ester obtained during transesterification show low differences with the chromatographic results as shown in figure $9 a$ using a pre-processing method because the correlation coefficient $\left(R^{2}\right)$ was 0.985 whereas the RMSEC and RMSEP were respectively $4.08 \%$ and $4.10 \%$.

Using experiments with an ethanol to oil molar ratio of 45.4 , the calibration plot for ethyl ester content (figure $9 b$ ) shows a good correlation of $\mathrm{R}^{2}=0.983$ using eight latent variables. More latent variables are used here because of the calibration and validation samples sets which are too small in particular for the lower ester contents, the position of the tube which may change when the transflectance probe is used and a part of the optical beam may be absorbed by the tube. The RMSEC and RMSEP were respectively $2.34 \%$ and $3.52 \%$. Comparatively to the batch reaction 


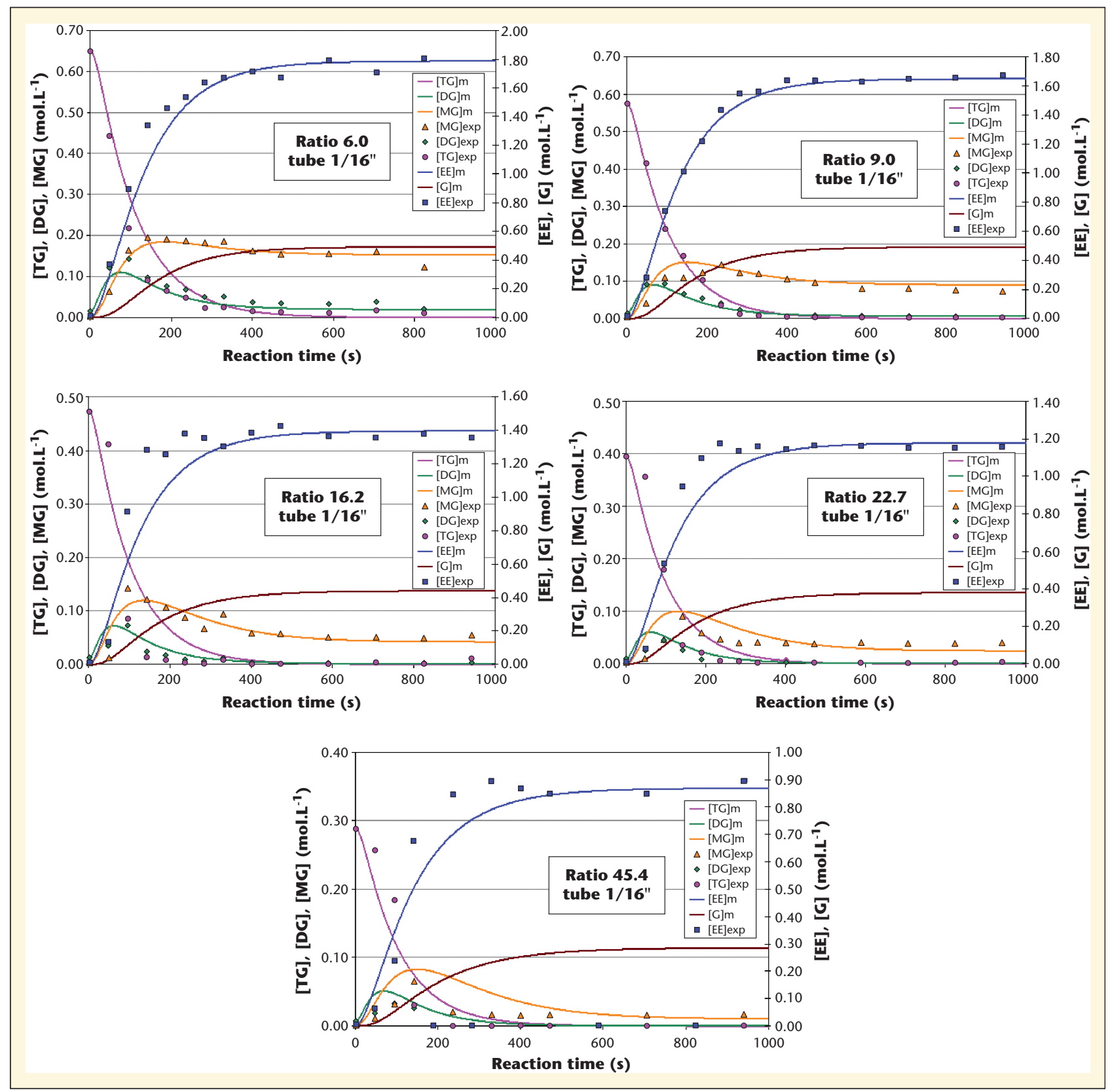

Figure 7. Modelling and experimental data representing the evolution of reaction medium composition according to the reaction time with the same set of parameters manually determined (valid for all molar ratios).

mixtures analyses performed in previous work (Richard et al., 2011; Richard et al., 2012a), the apparent standard errors of calibration increased because the NIR spectra obtained by the reflection probe or the transflectance probe and measured through PFA tube are noisier than the NIR spectra acquired with the transflectance optical probe directly dipped in the reactor (table 3 ). Indeed, the optical beam is altered when passing through the PFA tube due to dispersion. But these results are still satisfactory because the errors on calibration and prediction are lower than $5 \%$.

RMSEC and RMSEP are lower and then better in figure $9 b$ because the samples used were from the same ethanol to oil molar ratio (45.4); indeed the range of compositions of samples obtained from one molar ratio is more limited than the range of compositions of samples obtained for different molar ratios.

\section{Conclusion}

The high oleic sunflower oil transesterification reaction with ethanol catalyzed by EtONa has successfully been transposed from batch reactor to continuous 

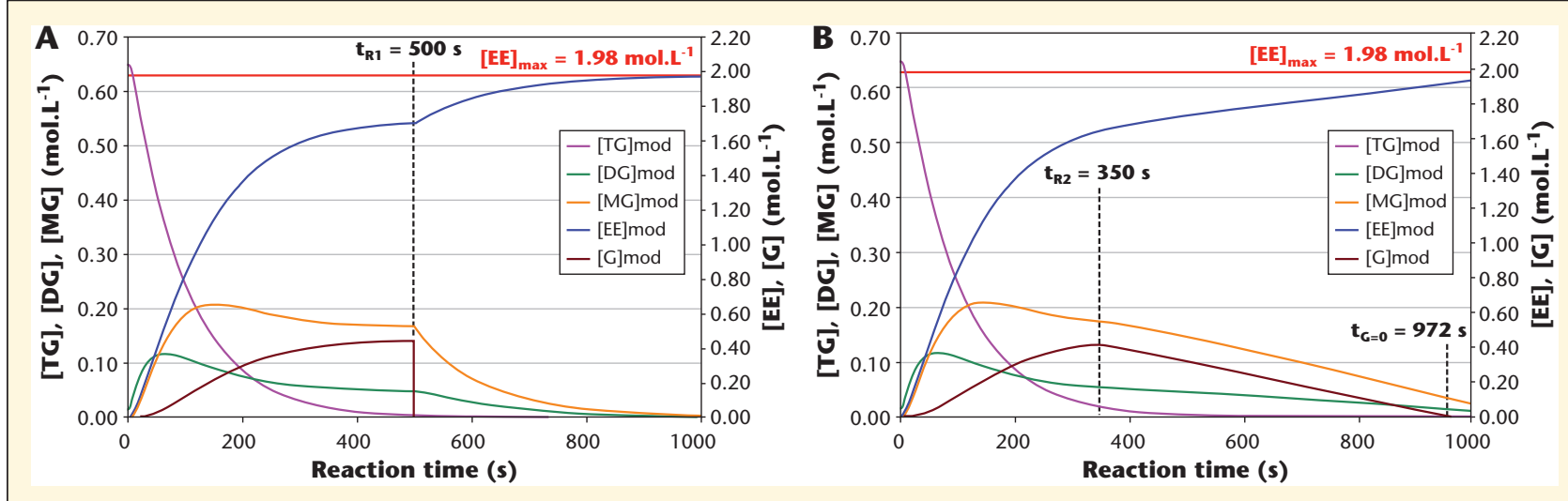

Figure 8. A) Simulation of a total glycerol removal from $t_{R 1}=500 \mathrm{~s}$ with $F_{G(R)}=0.5$ mol. $\mathrm{s}^{-1}$ during transesterification reaction. B) Simulation of constant and continuous glycerol removal from $t R 2=350 \mathrm{~s}$ with $F_{G(R)}^{\prime}=0.001 \mathrm{~mol} . \mathrm{s}^{-1}$ during transesterification reaction.
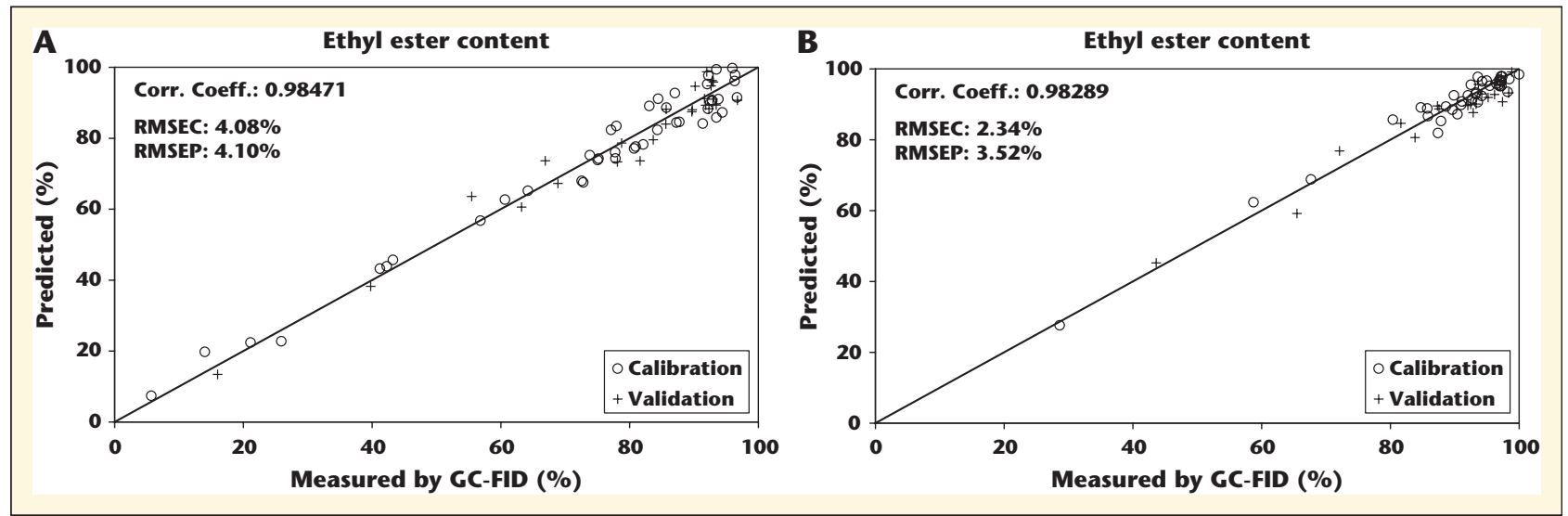

Figure 9. PLS regression of ethyl ester content of the reaction mixtures obtained $A$ ) with different ethanol to oil molar ratio at $65^{\circ} \mathrm{C}$ (data acquired with the reflection probe); $B$ ) with the same ethanol to oil molar ratio at $65^{\circ} \mathrm{C}$ (data acquired with the transflectance probe).

microreactor device. This study showed that, in the case of ethanolysis of high oleic sunflower oil, flow chemistry processes are an excellent way for obtaining more information in less time, more particularly at very low characteristic times. Moreover, two calibration models of NIR spectroscopy were developed to on-line evaluate the main component (ethyl ester) content during the transesterification reaction in microreactors. Results demonstrate that NIR spectroscopy is a fast and reliable technique to on-line determine the composition of a reaction mixture during transesterification without collecting and preparing samples. These data acquired at very low characteristic times enabled, thanks to a model, the representation of both phenomena: reaction kinetics and mass transfer. A global set of parameters is valid for all ethanol to oil molar ratios used. Finally, the model can be used to simulate other operating conditions such as the glycerol removal to shift the equilibrium towards product formation.

Table 3. Comparison of correlation in microreactor and batch reactor between NIR spectral data and GC-FID analysis.

\begin{tabular}{|c|c|c|c|c|}
\hline \multirow{2}{*}{ PLS model } & \multicolumn{2}{|l|}{ Microreactor } & \multicolumn{2}{|c|}{ 1L-batch reactor (Richard et al., 2011) } \\
\hline & $6<$ EtOH/oil $<45.4$ & Repeatability 45.4 & $\mathbf{3 0} \mathbf{C}<\mathbf{T}<\mathbf{7 0}^{\circ} \mathbf{C}$ & Repeatability $70^{\circ} \mathrm{C}$ \\
\hline Probe & Reflection & Transflectance & Transflectance & Transflectance \\
\hline $\mathrm{R}^{2}$ & 0.98471 & 0.98289 & 0.99361 & 0.98244 \\
\hline RMSEC & $4.08 \%$ & $2.34 \%$ & $1.26 \%$ & $0.38 \%$ \\
\hline RMSEP & $4.10 \%$ & $3.52 \%$ & $1.74 \%$ & $0.43 \%$ \\
\hline
\end{tabular}


Acknowledgements. We gratefully acknowledge the co-financial support from INRA (Institut National de la Recherche Agronomique) and the Région Midi-Pyrénées in France which enabled us to lead this research work in the Laboratoire de Chimie Agro-industrielle (LCA) and the Laboratoire de Génie Chimique (LGC).

\section{Disclosure}

Conflict of interest: none.

\section{REFERENCES}

Balabin RM, Safieva RZ, Lomakina EI. Gasoline classification using near infrared (NIR) spectroscopy data: Comparison of multivariate techniques. Anal Chim Acta 2010; 671: 27-35.

Chen T-Y, Chao C-S, Mong K-KT, Chen Y-C. Ultrasonication-assisted spray ionization mass spectrometry for on-line monitoring of organic reactions. Chem Commun 2010; 46: 8347-9.

Darnoko D, Cheryan M. Kinetics of palm oil transesterification in a batch reactor. / Am Oil Chem Soc 2000; 77: 1263-7.

Demirbas A, Karslioglu S. Biodiesel production facilities from vegetable oils and animal fats. Energy Sources Part A 2007; 29: 133-41.

Encinar JM, González JF, Rodríguez-Reinares A. Ethanolysis of used frying oil. Biodiesel preparation and characterization. Fuel Process Technol 2007; 88: 513-22.

Freedman B, Pryde EH, Mounts TL. Variables Affecting the Yields of Fatty Esters from Transesterified Vegetable-Oils. I Am Oil Chem Soc 1984; 61: 1638-43.

Fukuda H, Kondo A, Noda H. Biodiesel Fuel Production by Transesterification of Oils. Journal of Bioscience and Bioengineering 2001; 92: 405-16.
Guan G, Teshima M, Sato C, et al. Two-phase flow behavior in microtube reactors during biodiesel production from waste cooking oil. AIChE J 2010; 56: 1383-90.

Holcapek M, Jandera P, Fischer J, Prokes B. Analytical monitoring of the production of biodiesel by high-performance liquid chromatography with various detection methods. J Chromatogr A 1999; 858: 13-31.

Knothe G. Rapid monitoring of transesterification and assessing biodiesel fuel quality by near-infrared spectroscopy using a fiber-optic probe. J Am Oil Chem Soc 1999; 76: 795-800.

Knothe G. Analytical methods used in the production and fuel quality assessment of biodiesel. Trans ASAE 2001; 44: 193-200.

Mahamuni NN, Adewuyi YG. Fourier Transform Infrared Spectroscopy (FTIR) Method To Monitor Soy Biodiesel and Soybean Oil in Transesterification Reactions, Petrodiesel Biodiesel Blends, and Blend Adulteration with Soy Oil. Energy Fuels 2009; 23: 3773-82.

Meher LC, Vidya Sagar D, Naik SN. Technical aspects of biodiesel production by transesterification - a review. Renew Sust Energy Rev 2006; 10: 248-68.

Mittelbach M. Diesel Fuel Derived from Vegetable-Oils, V [1] - Gas-Chromatographic Determination of Free Glycerol in Transesterified Vegetable-Oils. Chromatographia 1993; 37: 623-6.

Poljanšek I, Likozar B, 2011. Influence of Mass Transfer and Kinetics on Biodiesel Production Process. In : Mass Transfer in Multiphase Systems and its Applications. M El-Amin. Ljubljana, InTech: 433-58.

Qiu Z, Zhao L, Weatherley L. Process intensification technologies in continuous biodiesel production. Chem Eng Process 2010; 49: 323-30.

Richard R, Dubreuil B, Prat L, Thiebaud-Roux $S$. On-line monitoring of the transesterification reaction carried out in microreactors using near infrared spectroscopy. Fuel 2012a; (in press) http://dx.doi.org/10.1016/j.fuel. 2012.07.054:

Richard R, Thiebaud-Roux S, Prat L. Modelling the kinetics of transesterification reaction of sunflower oil with ethanol in microreactors. Chemical Engineering Science 2012b (In press).

Richard R, Li Y, Dubreuil B, Thiebaud-Roux S, Prat L. On-line monitoring of the transesterification reaction between triglycerides and ethanol using near infrared spectroscopy combined with gas chromatography. Bioresour Technol 2011; 102: 6702-9.

Sharma YC, Singh B. Development of biodiesel from karanja, a tree found in rural India. Fuel 2008; 87: 1740-2.

Srivastava PK, Verma M. Methyl ester of karanja oil as an alternative renewable source energy. Fuel 2008; 87: 1673-7.

Stamenkovic OS, Veljkovic VB, Todorovic ZB, et al. Modeling the kinetics of calcium hydroxide catalyzed methanolysis of sunflower oil. Bioresour Technol 2011; 101: 4423-30.

Sun J, Ju J, Ji L, Zhang L, Xu N. Synthesis of Biodiesel in Capillary Microreactors. Ind Eng Chem Res 2008; 47: 1398-403.

Sun P, Wang B, Yao J, Zhang L, Xu N. Fast Synthesis of Biodiesel at High Throughput in Microstructured Reactors. Ind Eng Chem Res 2009; 49: 1259-64.

Trevisan MG, Garcia CM, Schuchardt U, Poppi RJ. Evolving factor analysis-based method for correcting monitoring delay in different batch runs for use with PLS: On-line monitoring of a transesterification reaction by ATR-FTIR. Talanta 2008; 74: 971-6.

Wen Z, Yu X, Tu S-T, Yan J, Dahlquist E. Intensification of biodiesel synthesis using zigzag micro-channel reactors. Bioresour Technol 2009; 100: 3054-60.

Zagonel GF, Peralta-Zamora P, Ramos LP. Multivariate monitoring of soybean oil ethanolysis by FTIR. Talanta 2004; 63: 1021-5. 